\title{
ON THE MEASURE-THEORETIC CHARACTER OF AN INVARIANT MEAN
}

\author{
R. G. DOUGLAS ${ }^{1}$
}

0. Introduction. M. M. Day has shown that an abelian group has only one invariant mean if, and only if, the group is finite [3, Theorem 4, p. 534]. Knowing this we now ask to what extent the invariant means on a given abelian group are alike. In this paper we shall show that they all have the same "measure-theoretic character."

An invariant mean on an abelian group $G$ is a normalized, positive, translation-invariant linear functional defined on the space of all bounded complex-valued functions on $G$. There is a canonical way of associating with the invariant mean $L$ a (unique) positive measure $\mu$ (which is invariant in a sense that will be made clear) defined on the Stone-Cech compactification of $G$ (discrete topology). Using this one-to-one correspondence, we shall ascribe to the invariant mean $L$ the measure-theoretic properties of $\mu$. Thus the measure algebra of the invariant mean $L$ is simply the measure algebra of the associated measure $\mu$. Our main theorem (Theorem 2) is that the measure algebras of any two invariant means on $G$ are isomorphic. A corollary extends this result to the measure algebras of invariant means on a subsemigroup of an abelian group.

An essential lemma in the proof of the main theorem is derived in \$2. We show that if $m$ is the cardinality of $G$ and is assumed to be infinite, then $2^{\mathfrak{m}}$ is the dimension of the $\mathfrak{R}_{2}$ space associated with an invariant mean on $G$. Because we believe this result to be of independent interest, we state it as Theorem 1.

A corollary to Theorem 1 gives a similar result for invariant means on certain abelian semigroups. In particular, this corollary is applicable to the semigroup of positive integers. An invariant mean on this semigroup is called a Banach mean. The corollary shows that the $\mathfrak{R}_{\mathbf{2}}$ space associated with a Banach mean is not separable. The question of the separability of this $\mathfrak{R}_{2}$ space was raised by P. Porcelli.

As a final topic we shall discuss (in $\$ 4$ ) some related problems. This discussion amounts to an exploration of further possibilities in the directions of Theorems 1 and 2, and we shall raise several questions in this connection.

Presented to the Society, January 26,1963, under the title $A$ uniqueness property of invariant means; received by the editors December 11, 1963 and, in revised forms, April 20, 1963 and August 15, 1963.

1 Research supported in part by a grant from the National Science Foundation. 
Now let us add a few comments before we begin. Firstly, a finitely additive invariant measure on $G$ is obtained by evaluating the invariant mean at the characteristic functions of subsets of $G$ (cf. $[6,17.22 \mathrm{a}$, pp. 242-243]). The countably additive measure that we associate with an invariant mean is the "Stone-Cech compactification" of this finitely additive measure (in the sense that their supporting spaces are so related), and we choose this measure because it is easier for our purposes to work with countably additive measures.

Secondly, the proof of the main theorem rests on Maharam's theorem on homogeneous measure algebras. Thus it is highly nonconstructive. A constructive proof, if one is possible, might yield more insight into the structure of invariant means.

The writer wishes to acknowledge his indebtedness to P. Porcelli for several very helpful conversations during the early stages of this paper and to thank M. Schreiber for his assistance in its final preparation.

1. Preliminaries. Let $\Sigma$ be an abelian semigroup. We shall denote by $\mathfrak{B}(\Sigma)$ the complex Banach space of bounded complex-valued functions on $\Sigma$ in which $\|f\|=\sup \{|f(\sigma)| \mid \sigma \in \Sigma\}$. An element $L$ of $\mathfrak{B}(\Sigma)^{*}$ (the conjugate space of $\mathfrak{B}(\Sigma)$ ) is said to be an invariant mean on $\Sigma$ if (1) $\|L\|=1$; (2) $L f \geqq 0$ for $f \geqq 0$; and (3) $L f_{\sigma}=L f$ for each $\sigma \in \Sigma$, where $f_{\sigma}(\tau)=f(\sigma+\tau)$ for $\tau \in \Sigma$. An abelian semigroup always has an invariant mean. The proof of this fact is due essentially to Banach, who proved it for certain special cases (see, e.g. [1, pp. 30-31]). For further information on invariant means see $[6, \$ 17]$.

Let $G$ be an abelian group. We shall denote by $\beta G$ the Stone-Cech compactification of the discrete space $G$. The complex Banach space $\mathfrak{S}(\beta G)$ of complex-valued continuous functions on $\beta G$ (with the supremum norm) is isometrically isomorphic to $\mathscr{B}(G)$. If $\pi$ is the inclusion map of $G$ into $\beta G$, then the induced map $\pi_{*}$ from $\mathbb{S}(\beta G)$ to $\mathfrak{B}(G)$ (for $f \in \mathfrak{S}(\beta G)$ and $\sigma \in G$ we have $\left(\pi_{*} f\right) \sigma=f(\pi \sigma)$ ) is an onto, isometrical isomorphism $[4,10.2$ and 10.3].

By the representation theorem of F. Riesz, we can identify $\mathbb{S}(\beta G)^{*}$ (conjugate space) with the space of finite complex-valued regular Borel measures on $\beta G$, which we denote by $M(\beta G)$. Further, let $\pi^{*}$ be the adjoint map of $\pi_{*}$, i.e., $\pi^{*}$ is the map defined from $\mathfrak{B}(G)^{*}$ to $\mathfrak{S}(\beta G)^{*}$ such that for $L \in \mathfrak{B}(G) *$ and $f \in \mathfrak{S}(\beta G)$ we have $\left(\pi^{*} L\right) f=L\left(\pi_{*} f\right)$. Then $\pi^{*}$ is an onto, isometrical isomorphism because $\pi_{*}$ is. Composing $\pi^{*}$ and the identification of $\mathfrak{S}(\beta G)^{*}$ and $\mathfrak{B}(G)^{*}$, we obtain the desired isometrical isomorphism from $\mathfrak{B}(G)$ * onto $M(\beta G)$. Let us denote the composite map by $\phi$. We can now define $\phi$ as follows: for $L \in \mathscr{B}(G)^{*}$, 
$\phi L$ is the unique measure $\mu \in M(\beta G)$ such that $\int_{\beta G} f d \mu=L\left(\pi_{*} f\right)$ for every $f \in \mathbb{S}(\beta G)$.

Corresponding to the group $\left\{T_{\sigma} \mid \sigma \in G\right\}$ of translation operators on $G\left(T_{\sigma} \tau=\sigma+\tau\right)$, there is the group $\left\{T_{\sigma}^{\prime} \mid \sigma \in G\right\}$ of homeomorphisms of $\beta G$, where each $T_{\sigma}^{\prime}$ is the unique extension of $T_{\sigma}$ [4, 0.12 and 6.5]. Let $U_{\sigma}$ denote the automorphism of $M(\beta G)$ induced by the measurable transformation $T_{\sigma}^{\prime}$, i.e., $\left(U_{\sigma} \mu\right)(E)=\mu\left(T_{\sigma}^{\prime}-1 E\right)$ for every Borel subset $E$ of $\beta G$. For any measure $\mu \in M(\beta G)$ a straightforward argument shows that $\phi^{-1} \mu$ is an invariant mean on $G$ if, and only if, $\mu$ is a probability measure (positive measure of mass one) that is invariant relative to the group of automorphisms $\mathcal{u}=\left\{U_{\sigma} \mid \sigma \in G\right\}$. We shall refer to such a measure as an invariant measure relative to $G$. Our problem now is to show that the measure algebras of two invariant measures are isomorphic.

2. Dimension of $\mathfrak{R}_{2}(\mu)$. We shall continue to use the notation and terminology of $\$ 1$ throughout the paper.

TheOREM 1. Let $G$ be an infinite abelian group of cardinality $\mathfrak{m}$ and $\mu$ an invariant measure relative to $G$. Then the dimension of $\mathfrak{R}_{2}(\mu)$ is $2^{\mathrm{m}}$.

Proof. A theorem of Kakutani [7, Theorem 1, p. 366] states that the cardinality of $\Gamma$, the abstract character group of $G$, is $2^{\mathfrak{m}}$. A character $\chi$ is a bounded complex-valued function on $G$; thus $\chi \in \mathscr{B}(G)$. Set $f_{x}=\pi_{*}^{-1} \chi$ and $\mathbf{X}=\left\{f_{\chi} \mid \chi \in \Gamma\right\}$; then $f_{\chi} \in \mathbb{S}(\beta G)$ and also $f_{\chi} \in \mathbb{R}_{2}(\mu)$. We shall prove that $X$ is an orthonormal subset of $\mathfrak{R}_{2}(\mu)$ and therefore that the dimension of $\ell_{2}(\mu)$ is at least $2^{\mathfrak{m}}$.

Let $V_{\sigma}$ be the linear operator induced on $\mathfrak{l}_{2}(\mu)$ by $T_{\sigma}^{\prime}$, i.e., $\left(V_{\sigma} f\right)(x)$ $=f\left(T_{\sigma}^{\prime} x\right)$ for $f \in \Omega_{2}(\mu)$ and $x \in \beta G$. It is clear that $V_{\sigma}$ is invertible because $V_{-\sigma}$ is its inverse. Further, for $f, g \in \mathbb{R}_{2}(\mu)$,

$$
\begin{aligned}
\left(V_{\sigma} f, V_{\sigma} g\right) & =\int_{\beta G} f\left(T_{\sigma}^{\prime} x\right) \bar{g}\left(T_{\sigma}^{\prime} x\right) d \mu(x)=\int_{\beta G} f(x) \bar{g}(x) d \mu\left(T_{\sigma}^{\prime-1} x\right) \\
& =\int_{\beta G} f(x) \bar{g}(x) d\left(U_{\sigma} \mu\right)(x)=(f, g), \quad \text { because } U_{\sigma} \mu=\mu .
\end{aligned}
$$

Hence $V_{\sigma}$ is a unitary operator. The following computation shows further that $V_{\sigma} f_{\chi}=\chi(\sigma) f_{\chi}$ :

$$
\begin{aligned}
{\left[\pi_{*}\left(V_{\sigma} f_{x}\right)\right](\tau) } & =\left(V_{\sigma} f_{x}\right)(\pi \tau)=f_{x}\left(T_{\sigma}^{\prime}(\pi \tau)\right)=f_{x}(\pi(\sigma+\tau)) \\
& =\left(\pi_{*} f_{x}\right)(\sigma+\tau)=\chi(\sigma+\tau)=\chi(\sigma)\left(\pi_{*} f_{x}\right)(\tau) .
\end{aligned}
$$

Thus $f_{\chi}$ is an eigenvector of $V_{\sigma}$ having the eigenvalue $\chi(\sigma)$. Now $G$ distinguishes the elements of $\Gamma$, i.e., for $\chi, \chi^{\prime} \in \Gamma, \chi \neq \chi^{\prime}$, there is a 
$\sigma \in G$ such that $\chi(\sigma) \neq \chi^{\prime}(\sigma)$. Therefore each pair $f_{\chi}$ and $f_{\chi^{\prime}}\left(f_{\chi^{\prime}} \neq f_{\chi^{\prime}}\right)$ is for some $\sigma \in G$ a pair of eigenvectors of the unitary operator $V_{\sigma}$ having distinct eigenvalues. Hence $\left(f_{\boldsymbol{x}}, f_{\mathbf{x}^{\prime}}\right)=0$ and $\mathbf{X}$ is an orthonormal subset of $\mathfrak{R}_{2}(\mu)$.

We shall now exhibit a dense subset of $\mathfrak{R}_{2}(\mu)$ having cardinality $2^{\mathfrak{m}}$, thus proving that the dimension of $\mathbb{R}_{2}(\mu)$ is exactly $2^{\mathfrak{m}}$. The set of all subsets of $G$ has cardinality $2^{\mathfrak{m}}$. For each subset $E$ of $G$ let $h_{\mathbb{z}}$ denote the characteristic function of $E$, i.e., $h_{E}(\sigma)$ is equal to $1, \sigma \in E$, or 0 , $\sigma \notin E$. Then the class of functions of the form $\sum_{i=1}^{n} \lambda_{i} h_{E_{i}}$, where each $\lambda_{i}$ is a complex number and each $E_{i}$ a subset of $G$, is a dense subspace of $\mathfrak{B}(G)$. (To see this, observe that for $f \in \mathfrak{B}(G)$ and $\epsilon>0$, there exists a finite partition $\left\{R_{i}\right\}_{i=1}^{n}$ of the range of $f$ such that

$$
\sup \left\{|f(\sigma)-f(\tau)| \mid \sigma, \tau \in f^{-1}\left(R_{i}\right)\right\}<\epsilon
$$

for $i=1,2, \cdots, n$. If we set $E_{i}=f^{-1}\left(R_{i}\right)$ and $\lambda_{i}=f\left(\sigma_{i}\right)$ for some $\sigma_{i} \in E_{i}$, then $\left.\left\|f-\sum_{i=1}^{n} \lambda_{i} h_{B_{i}}\right\|<\epsilon_{\text {. }}\right)$ Therefore the image of this class under $\pi_{*}$ is dense in $\mathfrak{S}(\beta G)$ and thus also in $\mathfrak{R}_{2}(\mu)$ [5, Problem 2, p. 242]. Hence the dimension of $\mathfrak{R}_{2}(\mu)$ is exactly $2^{\mathfrak{m}}$ and the theorem is proved.

A Banach mean is an invariant mean on the semigroup $Z^{+}$of positive integers. Let us carry out a construction paralleling that of $\S 1$ in which $G$ is replaced by $Z^{+}$. The positive integers are sufficient to distinguish the elements of the character group $T$ of the integers. Therefore the reasoning used in the proof of Theorem 1 (in which $V_{\sigma}$ is now shown to be an isometry and $f_{\chi}$ is now defined to be $\pi_{*}^{-1}\left(\chi^{+}\right)$, where $\chi^{+}$is $\chi \in T$ restricted to $Z^{+}$) is applicable in this context. Moreover, this reasoning is applicable in a more general context of which this is a special case. The following corollary summarizes these remarks.

CoROllary. Let $\Sigma$ be a subsemigroup of the infinite abelian group $G$ of cardinality $\mathrm{m}$ and let $\mu$ be an invariant measure relative to $\Sigma$. Assume further that $\Sigma$ distinguishes the characters of $G$. Then the dimension of $\mathfrak{R}_{2}(\mu)$ is $2^{\mathfrak{m}}$.

3. Uniqueness of the measure algebra. For any measure algebra $B$ let $K(B)$ denote the smallest cardinal of a sigma-basis for $B$. $B$ is said to be homogeneous if $K(B)=K(P)$ for every (principal) ideal $P \subseteq B$ different from the null ideal. The theorem of Maharam [8, Theorem 1, p. 109] immediately implies that if $B$ is homogeneous and nonatomic, then $B$ is determined within an (measure-preserving) isomorphism by $K(B)$. Moreover, if $B$ is homogeneous and $K(B)$ is infinite, it is easy to see that $B$ must be nonatomic. Thus an infinite 
homogeneous measure algebra $B$ is determined within an (measurepreserving) isomorphism by $K(B)$. This is the form of Maharam's theorem which we shall use.

THEOREM 2. The measure algebras of two invariant measures relative to $G$ are isomorphic.

Proof. If $G$ is finite, the theorem follows from the uniqueness of the invariant mean. Assume therefore that $G$ is an infinite abelian group having cardinality $\mathfrak{m}$ and that $\mu$ is an invariant measure relative to $G$. We shall show that the measure algebra $B_{\mu}$ of $\mu$ is homogeneous and that $K\left(B_{\mu}\right)=2^{\mathfrak{m}}$. An application of the aforementioned form of Maharam's theorem will then complete the proof.

First, the dimension of $\mathfrak{R}_{2}(\mu)$ is equal to $K\left(B_{\mu}\right)$ (cf. $[6,16.12$, p. 225]). (The assertion of [6] is not immediately applicable in the context of [8] because of a difference in the meanings of (sigma) "basis." However, the reader can easily verify that for any infinite measure algebra the smallest cardinals of a basis in the senses of [6] and [8], respectively, are equal.) Therefore it follows from Theorem 1 that $K\left(B_{\mu}\right)=2^{\mathfrak{m}}$.

Secondly, suppose $B_{\mu}$ is not homogeneous. Then the unit $e$ of $B_{\mu}$ can be decomposed such that: (1) $e=x \bigvee x^{\prime}, x \wedge x^{\prime}=0$ and $x \neq 0$, (2) for $P_{x}$, the principal ideal of $B_{\mu}$ generated by $x, K\left(P_{x}\right)<2^{\mathfrak{m}}$, and (3) $K\left(P_{y}\right)>K\left(P_{x}\right)$ for $0<y \leqq x^{\prime}\left[8\right.$, Theorem 2, p. 111]. Let $W_{\sigma}$ be the automorphism of $B_{\mu}$ induced by $T_{\sigma}^{\prime}$. We shall show that $x$ is invariant relative to each $W_{\sigma}$ and hence that "the part of $\mu$ supported on $x^{\prime \prime}$ is invariant relative to $\mathcal{u}$.

Let $W_{\sigma} x=z \bigvee z^{\prime}$, where $0 \leqq z \leqq x$ and $0 \leqq z^{\prime} \leqq x^{\prime}$. Because $W_{\sigma}$ is an automorphism, $K\left(P_{x}\right)=K\left(P_{W_{\sigma} x}\right) \geqq K\left(P_{z^{\prime}}\right)$. However, it follows from (3) that if $z^{\prime} \neq 0$, then $K\left(P_{z^{\prime}}\right)>K\left(P_{x}\right)$. Therefore $z^{\prime}=0$ and $W_{\sigma} x \leqq x$. But $W_{\sigma}$ is measure-preserving and thus $W_{\sigma} x=x$.

Let $F$ be a Borel subset of $\beta G$ that corresponds to $x$ and let $v$ be the measure in $M(\beta G)$ defined such that $v(E)=\mu(E \cap F)$ for each Borel subset $E$ of $\beta G$. Then $[\mu(F)]^{-1} v$ is a probability measure and moreover is invariant relative to $\mathcal{U}$ because $x$ is invariant relative to each "translation" $W_{\sigma}$. Therefore the dimension of $\mathfrak{l}_{2}(v)$ is $2^{\mathfrak{m}}$ by Theorem 1 and also $K\left(B_{v}\right)=2^{\mathfrak{m}}$. But $B_{v}$ and $P_{x}$ are isomorphic measure algebras and hence $K\left(P_{x}\right)=2^{\mathfrak{m}}$. This contradicts (2); therefore $B_{\mu}$ is homogeneous and the theorem is proved.

Corollary. Let $\Sigma$ be a subsemigroup of the abelian group $G$. The measure algebras of two invariant measures relative to $\Sigma$ are isomorphic.

Proof. If $\Sigma$ is finite, it is easy to see that the invariant mean on 
such an abelian semigroup is unique. Therefore assume $\Sigma$ is infinite. If $H=\left\{\sigma \tau^{-1} \mid \sigma, \tau \in \Sigma\right\}$, then $H$ is the subgroup of $G$ generated by $\Sigma$. Clearly $\Sigma$ distinguishes the characters of $H$. Thus the corollary to Theorem 1 is applicable to $\Sigma$ considered as a subsemigroup of $H$. If we now assume that the construction outlined in $\$ 1$ has been carried out for $\Sigma$, we can repeat in this context the argument (we replace Theorem 1 by the corollary) used for the infinite case in the proof of Theorem 2. This completes the proof of the corollary.

4. Further problems. In the proof of Theorem 1, we saw that $X$ is an orthonormal subset of $\mathfrak{R}_{2}(\mu)$. Moreover, the cardinality of $\mathbf{X}$ is equal to the dimension of $R_{2}(\mu)$. A natural question is whether or not $\mathrm{X}$ is complete in $\mathbb{R}_{2}(\mu)$.

Let $\mathscr{I}$ denote the set of all invariant measures relative to $G$; then $g$ is a $w^{*}$-compact, convex subset of $M(\beta G)$. Therefore $g$ is the $w^{*}$ closed convex hull of its extreme points, by the Kreln-Mil'man Theorem. An extreme point of $g$ will be referred to as an elementary invariant measure relative to $G$.

Now if $\mu$ is not elementary, $\mathrm{X}$ is not complete. Take $\mu=\left(v+v^{\prime}\right) / 2$ with $v, v^{\prime} \in g$ and $v \neq v^{\prime}$. Then the Radon-Nikodym derivative of $v-v^{\prime}$ is in $\ell_{2}(\mu)$ but is orthogonal to $X$.

If $\mu$ is elementary, we do not know the answer and so our first question is: if $\mu$ is an elementary invariant measure relative to $G$, is $\mathbf{X}$ complete in $\mathfrak{R}_{2}(\mu)$ ? A second related question is: does the completeness of $\mathrm{X}$ depend only on the group $G$ or does it, perhaps, also depend on which elementary invariant measure is chosen?

Note that if for some $G$ and $\mu, \mathrm{X}$ were complete in $\mathfrak{R}_{2}(\mu)$, the measure algebra of $\mu$ would be isomorphic to that of the Haar measure on the (maximal) Bohr compactification of $G[6,26.11$, p. 430]. Moreover, this isomorphism would preserve the action of the automorphism group of "translations" on the measure algebras.

Let us now observe that the group of automorphisms which act on the measure algebra of an invariant mean was not mentioned in the statement of the main theorem. It would be desirable to know that the isomorphism (whose existence is stated in the main theorem) could be effected in such a way as to preserve the action of this automorphism group. This is not possible in general for a rather apparent reason. If the action of the automorphism group is ergodic on the first measure algebra and not on the second, then such an isomorphism is clearly impossible. It can be shown that the action of the automorphism group on the measure algebra of an invariant mean is ergodic if, and only if, the associated invariant measure is elementary (cf. 
[2, Theorem 2, p. 1126]). Thus we state our final question as follows: does there exist an isomorphism between the measure algebras of two elementary invariant measures that preserves the action of the automorphism group?

Added in proof. If

$$
\mathcal{J}=\left\{\nu \in M(\beta G) \mid \nu \geqq 0, \nu(\beta G)=1 \text { and } \int_{\beta G} \chi d \nu=0 \text { for } 1 \neq \chi \in \mathrm{X}\right\}
$$

then $\mathcal{I}$ is a $w^{*}$-compact convex subset of $M(\beta G)$ that contains $\mathcal{g}$. It follows from a recent result of ours [External measures and subspace density, Michigan Math. J. 11 (1964), 243-246] that X spans $\mathfrak{R}_{2}(\nu)$ if, and only if, $\nu$ is an extreme point of $\mathcal{J}$. Thus our first question can be reformulated to ask whether each extreme point of $g$ is also an extreme point of $\mathcal{g}$.

\section{BIBLIOGRAPHY}

1. S. Banach, Theorie des operations linéaires, Monogr. Mat., Warsaw, 1932.

2. J. R. Blum and D. L. Hanson, On invariant probability measures. I, Pacific J. Math. 10 (1960), 1125-1129.

3. M. M. Day, Amenable semigroups, Illinois J. Math. 1 (1957), 509-544.

4. L. Gillman and M. Jerison, Rings of continuous functions, Van Nostrand, New York, 1960.

5. P. R. Halmos, Measure theory, Van Nostrand, New York, 1950.

6. E. Hewitt and K. A. Ross, Abstract harmonic analysis. I, Grundlehren der Mathematischen Wissenschaften, Bd. 115, Springer, Berlin, 1963.

7. S. Kakutani, On cardinal numbers related with a compact abelian group, Proc. Imp. Acad. Tokyo 19 (1943), 366-372.

8. D. Maharam, On homogeneous measure algebras, Proc. Nat. Acad. Sci. U. S. A. 28 (1942), 108-111.

Louisiana State UnIversity aND UNIVERSITY OF MICHIGAN 